\title{
超高强钢辊弯成形回弹机理分析及控制研究
}

\author{
韩飞牛丽丽王允杨亚辉 \\ (北方工业大学机械与材料工程学院 北京 100144)
}

\begin{abstract}
摘要: 辊弯成形技术能够生产高强度、性能佳、高精度的型钢产品, 并且具有高效、节能、省材等特点, 在许多领域得到广泛运 用。针对高强钢辊弯成形后出现回弹现象, 设计两种轧辊设计方案, 建立 ABAQUS 有限元模型, 分析不同设计方案下板材在成形 过程中的等效塑性应变和等效应力的分布情况, 重点阐述辊弯成形件回弹的成形机理, 提出控制回弹的成形工艺方法; 试验验证 轧辊设计优化后工件回弹角度更小, 成形精度更好, 回弹角度最大误差为 $1.66 \%$; 通过仿真分析, 轧辊设计优化后的成形件边部纵 向应变值降低并趋于平缓，结果表明采用优化后的工艺参数可有效减小板材的冚余变形，并有效控制板材的回弹问题。
\end{abstract}

关键词: 辊弯成形; 轧辊设计; 数值模拟; 回弹控制

中图分类号: TH16

\section{Research on Springback Mechanism and Control of Ultra High Strength Steel in Roll Forming Process}

\author{
HAN Fei NIU Lili WANG Yun YANG Yahui
}

(School of Mechanical and Materials Engineering, North China University of Technology, Beijing 100144)

\begin{abstract}
Roll forming technology can obtain better products which have high strength, better performance and high precision and has been widely used in many fields. This paper focuses on research of springback control of ultra high strength steel, and investigate two different roller design. The finite element is built to analysis the distribution of equivalent plastic strain and equivalent stress value , and the springback mechanism of roll forming is expounded and a process methods to improve the springback is proposed. The result from the simulations and experiments show that the springback angle of part is smaller after optimizing the rollers, and the sectional dimension is better as well. The maximum error of springback angle is $1.66 \%$. It shows that the springback angle have been controlled effectively. The simulation results show that the longitudinal strain value of the edge is reduced and tends to be smooth and the optimized process parameters can effectively reduce the redundant deformation and control the springback of the sheet.
\end{abstract}

Key words: roll forming; roller design; numerical simulation; springback control

\section{0 前言}

板材成形过程中普遍存在回弹问题，特别是高 强钢的弯曲过程中回弹现象更为严重, 对零件的尺 寸精度造成很大的影响, 随着汽车轻量化进程中先 进高强钢材料的应用, 成形件的回弹问题一直是行 业关注的热点问题，对成形件的回弹控制和回弹机 理的研究具有实际意义。辊弯成形是将金属板材通 过连续多道次具有特定截面轮廓的轧辊, 横向弯曲 成特定截面的一种方法 ${ }^{[1]}$ 。采用辊弯成形工艺加工 的成形件具有较好的截面力学性能和截型尺寸。

* 国家自然科学基金(50905001, 51074204)、北京市自然科学基金(3112010) 和北京市青年拔尖人才 $(2014000026833 Z$ K12)资助项目。20170503 收到 初稿, 20170904 收到修改稿
PANTHI 等 ${ }^{[2-3]}$ 以材料特性为研究对象, 对比了 不同的材料力学性能和几何参数以及时间对回弹量 的影响, 阐述了板材的回弹机理; XI 等 ${ }^{[4-6]}$ 基于经 典弯曲理论开发程序用于更新的模型, 试验和预测 之间的结果表明, 经验公式中对于参数的精确选取 能够提高高强钢回弹预测精度。BUI 等 ${ }^{[7]}$ 以成形工 艺参数为研究对象, 分析板带在变形过程中材料的 位移轨迹与纵向应变, 探讨了道次间距、成形速度 和摩擦对于回弹量的影响。WEIBENGA 等 ${ }^{[8]}$ 在研究 工艺缺陷纵向弓以及回弹问题的基础上, 结合优化 技术, 建立目标函数, 确定关键参数在成形过程中 最优解, 修正和优化了辊弯成形工艺参数, 并确定 高强钢材料产品道次间相关参数的限制范围, 这些 结论对实际生产起到有效的指导作用。JOHN 等 ${ }^{[9]}$ 通过设计下山量, 研究此方法对生产 $\mathrm{V}$ 形截面辊弯 
产品的质量影响的研究。解析计算不同辊花图下 V 形截面产品用下山法后, 得出产品边缘应力应变计 算公式, 并通过模拟试验论证了下山法可以降低产 品翼缘纵向应变值, 从而可以减小产品纵向应变缺 陷。SAFDARIAN 等 ${ }^{[10]}$ 研究了 $U$ 形件辊弯成形部 分道次成形过程中成形参数对纵向应变和纵向弓 缺陷的影响。张宝等 ${ }^{[11]}$ 采用下山法设计轧辊, 通过 试验和仿真结果表明, 成形件的纵向拉伸比传统轧 辊设计有减小, 说明轧辊设计能有效减少板材残余 应力和圥余变形。随着汽车、建筑行业对预切口截 面产品需求的增加, 研究如何尽可能减小预冲孔辊 弯产品的缺陷问题, 是当下辊弯成形工艺研究的热 点。伊朗学者 BIDABADI 等 ${ }^{[12-13]}$ 研究了传统辊弯 工艺参数, 如辊花类型、道次间距、预冲孔直径, 孔的纵向距离等对最终产品精度的影响。结果表 明: 弯角增量依然是影响产品精度的重要因素, 此外腹板宽度、润滑条件等也是相关性较强的因素 之一。

本文研究辊弯成形的回弹机理分析及控制, 针 对下弯角回弹提出两种控制回弹的轧辊设计方案, 通过分析下弯角区板材应力应变的分布, 阐述辊弯 成形回弹的产生特点; 通过试验验证了仿真模型的 计算精度, 在回弹成形机理分析上进行轧辊设计, 实现了成形件的回弹补偿; 对比两种轧辊设计方案 下, 弯角区及翼缘区单元的纵向应变, 结果表明轧 辊优化后产品的成形精度得到提高, 为辊弯成形件 的回弹控制研究提供参考。

\section{1 试验设计及模型建立}

\section{1 试验方法}

设计帽型件目标截型及辊花图如图 1 所示, 成 形材料选取汽车用先进超高强度钢, 充分考虑材料 强度大易发生回弹的特点, 用定长度法对每个道次 的弯角半径进行分配; 成形试验在北方工业大学高 精度辊弯成形试验机上进行, 如图 2 所示, 道次间 距 $250 \mathrm{~mm}$, 单电动机下辊驱动。

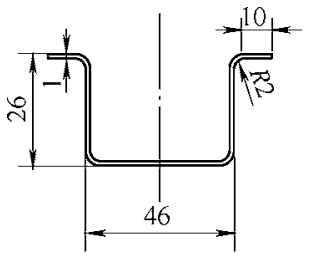

(a) 成形件截形尺寸

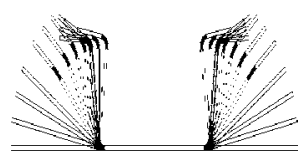

(b) 轧辊设计辊花图
图 1 成形截面与辊花图

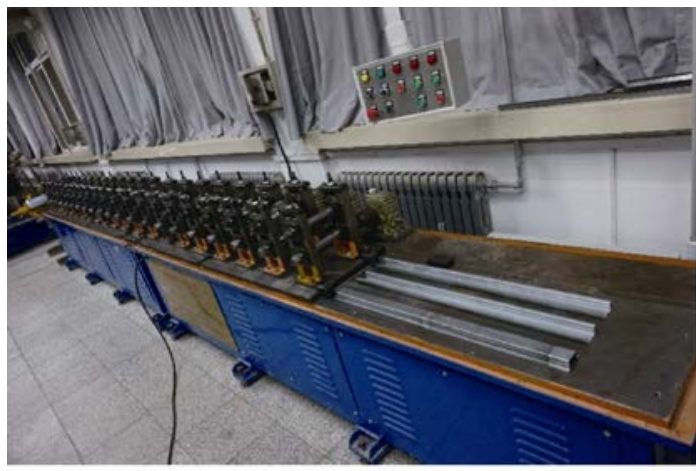

图 2 辊弯成形试验机

为准确描述成形工件弯角处的回弹, 如图 3 是 测量回弹角度的示意图。 $\theta$ 表示成形件的下弯角回 弹角, $\delta$ 表示成形件的上弯角回弹角。

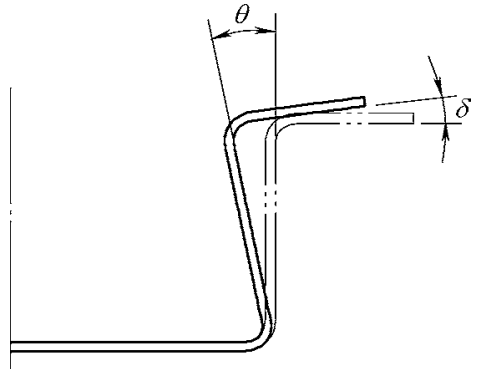

图 3 回弹角示意图

\section{2 有限元建模}

建立有限元仿真模型如图 4 所示，采用 Abaqus/Explicit 对成形过程进行仿真，选用的是减 缩积分实体单元，为尽可能模拟实际成形工况，板 材边界约束仅限制沿板厚轴向的转动、以及沿横向 弯曲方向的平动, 其他自由。

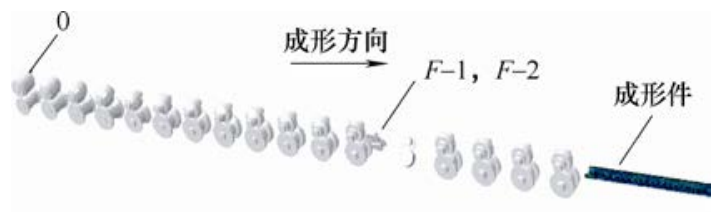

图 4 辊弯成形仿真模型

将板材送入第一道次, 轧辊以恒定的速度转动, 板材发生变形后将与轧辊间产生正压力, 由轧辊转 动时压力附加的摩擦力带动板带前进，其中 F-1、 $\mathrm{F}-2$ 是左右两个辅辊, 成形板材采用 $\mathrm{Q} \& \mathrm{P}$ 钢, 板材 变形区网格细化, 初始设计板材长度为 $900 \mathrm{~mm}$, 板 宽为 $115.6 \mathrm{~mm}$ 。

为得到本试验当中的材料模型, 首先通过单向 拉伸试验获得工程应力-工程应变数据, 已知材料在 拉伸过程中，横截面不断变化，通过解析计算求得 真实应力-真实应变数据, 如图 5a 所示。

仿真模型要尽可能与真实材料相近，因此材料 
属性输入采用拉伸试验所得到的优化后的数据模 型, 如图 5b 所示, 在缩颈点之后, 材料出现孔洞、 撕裂应力状态已经发生变化, 在缩颈点之后的数据 代入到有限元模拟当中不准确, 因此常采用拟合曲 线对缩颈点之后曲线进行修正。本文采用的是 Swift 修正式, 即

$$
\sigma=A \varepsilon^{n}+C
$$

式中, $A 、 n 、 C$ 为待定参数。

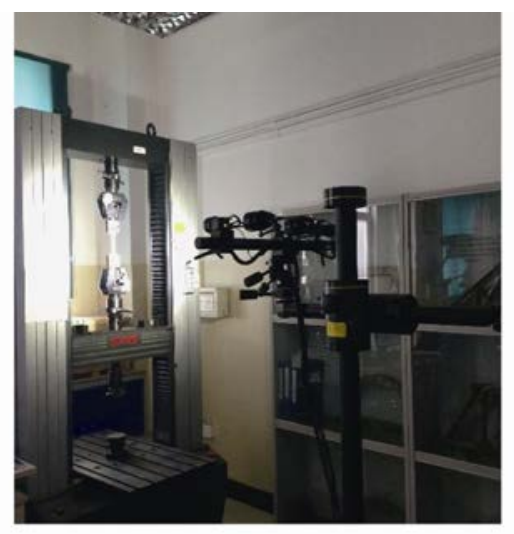

(a) 单向拉伸试验平台

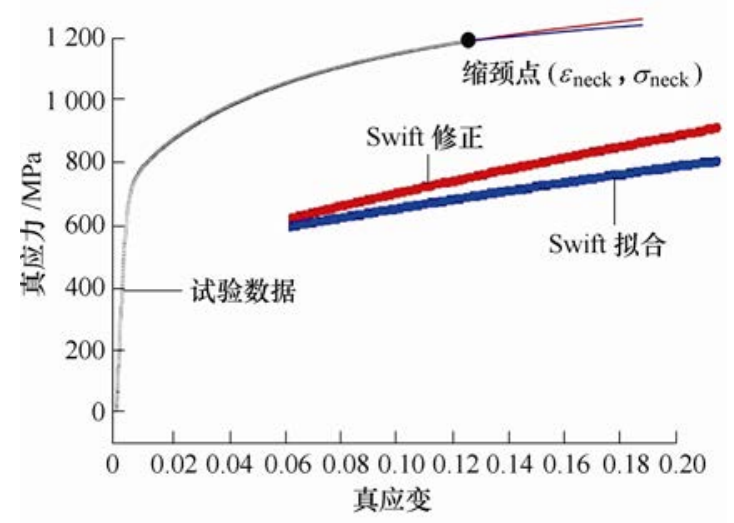

(b) 材料应力应变曲线

图 5 试验平台与应力应变曲线

考虑到重点研究板材弯曲部位的应力应变分 布, 将板材沿横向划分为翼缘、上弯角、下弯角、 腹板、立边, 其中上、下弯角将网格细化; 厚度方 向, 将板材分为上、下两层, 见图 6。

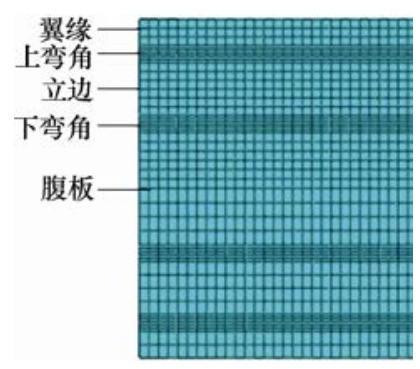

图 6 网格划分示意

对于下弯角过弯道次, 为使板材内外层与轧辊 充分接触, 对辅助辊设计做了优化修改, 前后辅辊
如图 7 所示。

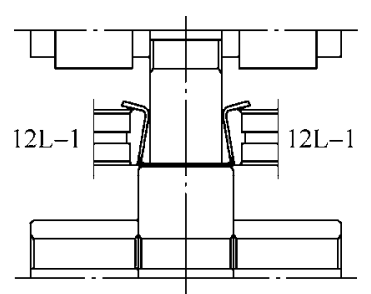

(a) 方案 1( 修改辅辊前)

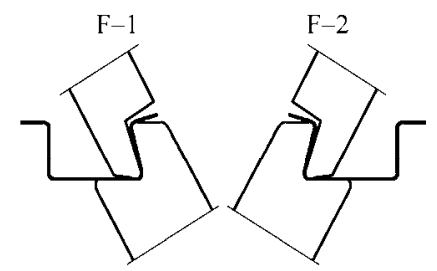

(b) 方案 2 (修改辅辊后)
图 7 过弯道次轧辊设计

\section{2 有限元模型精度验证}

将成形完成的帽型件与仿真结果对比, 验证仿 真模型的准确性。提取方案 2(修改辅辊后)的成形件 端面 $(Z=450 \mathrm{~mm}$ 处)上的关键点, 导入坐标系与仿真 截面对比, 结果如图 8 和表 $1 、 2$ 所示。

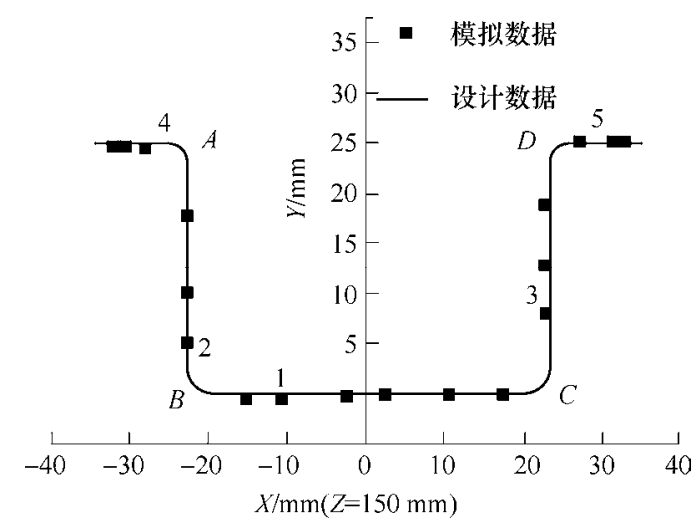

图 8 试验采点和模拟截形对比

\section{表 1 参考点 $X / Y$ 偏移误差}

\begin{tabular}{cccccc}
\hline & \multicolumn{5}{c}{ 参考点编号 } \\
\cline { 2 - 6 } & 1 & 2 & 3 & 4 & 5 \\
\hline$Y$ 向模拟结果 $/ \mathrm{mm}$ & 0.16 & 4.76 & 8.70 & 24.80 & 24.89 \\
$Y$ 向实测结果 $/ \mathrm{mm}$ & 0.09 & 4.99 & 8.00 & 24.34 & 24.32 \\
误差(实测值-模拟值) & -0.05 & 0.23 & -0.70 & -0.46 & -0.57 \\
\hline$X$ 向模拟结果 $/ \mathrm{mm}$ & -11.41 & -21.35 & 22.56 & -24.36 & 28.00 \\
$X$ 向实测结果 $/ \mathrm{mm}$ & -11.00 & -23.00 & 23.66 & -27.99 & 29.49 \\
误差(实测值-模拟值) & 0.41 & -1.65 & 1.10 & -3.63 & 1.49 \\
\hline
\end{tabular}

表 2 弯角处成形角度误差对比

\begin{tabular}{ccccc}
\hline & \multicolumn{4}{c}{ 成形角度 } \\
\cline { 2 - 5 } & $A$ & $B$ & $C$ & $D$ \\
\hline 模拟结果 $/\left(^{\circ}\right)$ & 98.45 & 98.54 & 98.38 & 96.37 \\
误差 $\left(\Delta / 90^{\circ}\right)(\%)$ & 9.39 & 9.39 & 9.31 & 7.08 \\
\hline 实测结果 $/\left(^{\circ}\right)$ & 89.69 & 90.32 & 91.49 & 90.67 \\
误差 $\left(\Delta / 90^{\circ}\right)(\%)$ & -0.34 & 0.35 & 1.66 & 0.74 \\
\hline
\end{tabular}

注: 误差 $\Delta / 90^{\circ}$ 表示模拟或实测值减去 $90^{\circ}$ 后与 $90^{\circ}$ 的比值。

通过仿真截面形状与辊弯成形试验截面形状的 对比结果, 可知端面尺寸与模拟结果的截面尺寸吻 合程度较好, 仿真结果与实际结果的尺寸误差较小, 
建立的帽型件辊弯成形工艺有限元仿真模型能够 有效地模拟成形过程。

\section{3 试验结果及分析}

\section{1 成形件回弹机理分析}

辊弯成形是典型的增量成形工艺, 成形过程中, 轧辊与板材之间的摩擦带动板材向前运动, 板材在 轧辊作用下发生弹性、塑性变形。当板材离开轧辊 后, 弹性变形恢复, 弹性能被释放, 塑性变形被保 留, 在此阶段板材易发生回弹。因此, 辊弯成形过 程也可看作板材进行反复加载-卸载的受力过程, 是 通过多次小变形累积而达到最终变形量。

图 9 是提取方案 1 和方案 2 仿真模型板材的左 下弯角处单元的 Mises 应力和等效塑性应变。可知 随着成形进行, 每一次的应变增量都是因为与轧辊 接触的而发生塑性变形, 板材离开轧辊后, 该单元 节点卸去外载荷, 在下一道次中, 重新加载进入塑 性变形, 因此, 板材的应变值在逐道次累加, 而应 力值发生阶段性回复, 单元节点处发生相应的弹性 回复，而塑性应变在不断累积。

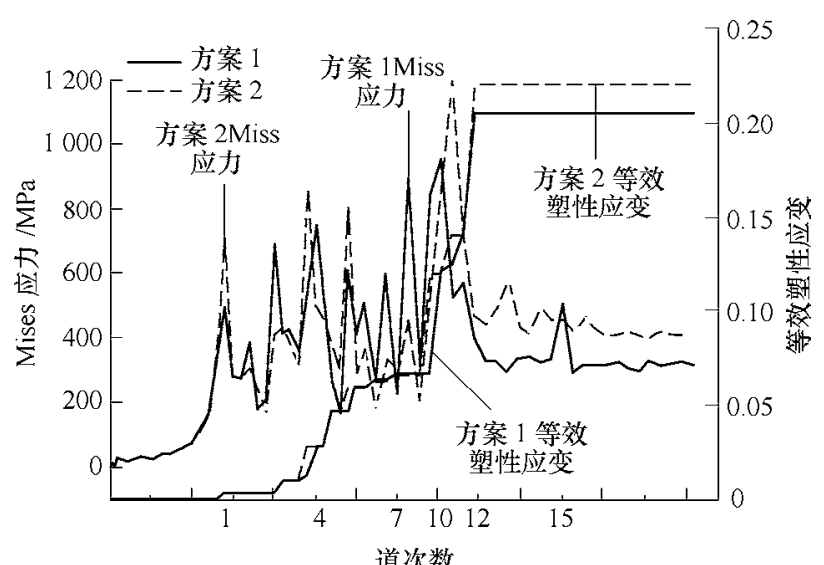

图 9 方案 1 和方案 2 历程等效应力应变对比

辊弯成形过程中，在板材接触轧辊时等效应力 迅速上升并达到一个峰值, 但当板材逐渐偏离轧辊 时, 等效应力随之降低, 证明了辊弯成形过程中就 存在残余应力的释放, 这也是辊弯成形的工艺特点 决定的, 每一道次的成形过程都是板材内部应力不 断重新平衡的过程, 所以成形过程也是残余应力不 断释放的过程。而该工艺的回弹是整个变形过程积 累下来的历史效应, 它的能量来自于在每一步成形 过程中所储存的弹性变形能, 在板材成形结束时, 随着载荷的释放或消失, 成形过程中所积累的弹性 变形能要释放出来, 导致零件整体形状发生改变, 最终导致零件成形后截型发生改变。在成形过程中,
整个系统的等效塑性应变呈现渐进阶梯形上升的形 态，正符合辊弯成形的工艺特点，证实了辊弯成形 是在成形过程中就出现弹性回复的特点。

对比两种方案下等效塑性应变的值, 可以看出 方案 2 下板材积累的等效塑性应变值更高。为了使 成形件塑性变形更完善, 成形精度更准确, 通常在 轧辊设计中增加内外实弯辅助辊道次, 用以提高板 材的塑性应变值, 从而起到补偿回弹的作用。

\section{2 成形件弯角处材料流动分析}

材料的薄厚能够反映成形过程中材料的流动。 图 10 为板材横向路径上过弯之后的厚度变化。

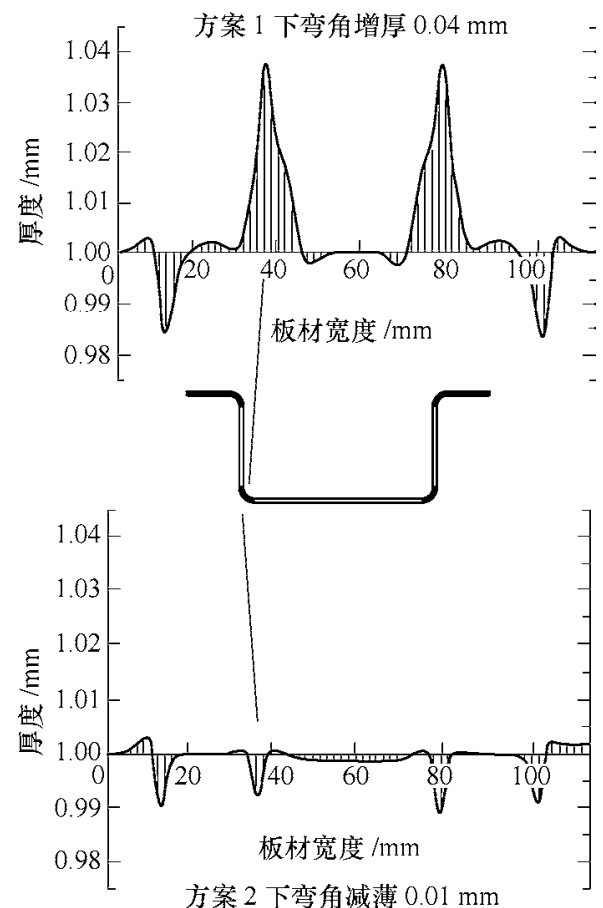

图 10 空弯和实弯对成形件弯角处厚度影响

方案 1 采用空管, 下弯角处的过弯对板材造成 $Z$ 方向的载荷, 而腹板处有下辊支撑且两侧对称, 板材无法发生 $Z$ 方向的位移, 故受压的材料流动到 下弯角处, 并且由于下弯角内侧存在空弯, 导致下 弯角处材料变厚。

为避免产生空弯, 方案 2 采用实弯使下弯角处 的板材内外侧均与轧辊充分接触, 弯角处材料减薄, 材料从增厚 $0.04 \mathrm{~mm}$ 至减薄 $0.01 \mathrm{~mm}$, 下弯角处的 变厚情况得到明显改善, 材料流动更为合理; 减薄 部位的幅值比修改辊型前下降, 且整体波动较小, 说明辊型修改后添加的辅助辊对弯角增厚能够起到 较明显的改善效果。

\section{3 成形件弯角处单元应力状态}

如表 3 所示两种回弹控制方式中左下弯角单元 应力状态。方案 1 过弯时的空弯造成了横向和纵向 的受压，导致了材料在下弯角处的堆积; 方案 2 在 
辊轮的压力及带动下, 单元的横向及纵向产生拉应 力, 材料有轻微的减薄趋势, 塑性变形区增大, 单 元格变形情况见图 11。

\begin{tabular}{ccc} 
表 3 & 下弯角单元 $\boldsymbol{Y} / \boldsymbol{Z}$ 向正应力对比 & $\mathrm{MPa}$ \\
\hline 参考点应力 & 方案 1 & 方案 2 \\
\hline$\sigma_{x}$ & -65.59 & 189.28 \\
$\sigma_{y}$ & -300.80 & 157.46 \\
\hline
\end{tabular}
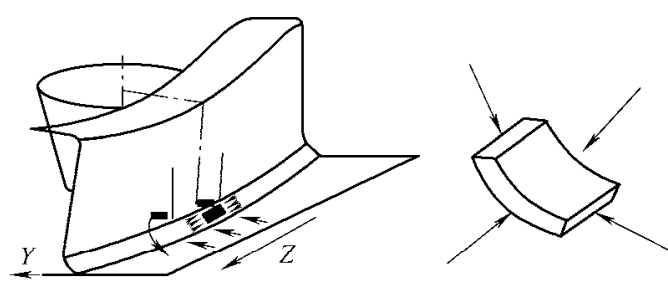

(a) 方案 1 (上) 弯角处应力状态
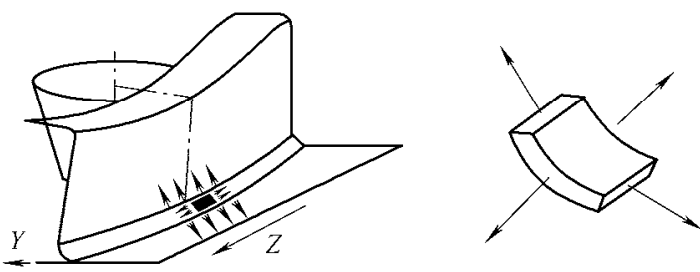

(b) 方案 2(下) 弯角处应力状态图

图 11 两种方案弯角处应力状态

\section{4 成形件在过弯道次的纵向应变历程分析}

除了弯角部位的应变集中改善外, 板材整体发生 的最大纵向应变也有一定程度的降低, 纵向应变属于 咒余变形, 值越小越有利于控制产品的质量, 设置辅 助辊前后, 板材的最大纵向应变值对比如图 12 所示。 分别提取成形过程中过弯道次及整形道次的纵向应 变的横向分布, 如图 13a、13b 所示, 方案 1 在经历 过弯阶段时, 下弯角部位发生较大的纵向应变激变, 由于塑性变形少, 回弹量较大。方案 2 对下弯角进行 逐次过弯, 下弯角处的受压状况得到明显改善, 纵向 应变变化趋于平缓, 且最大纵向应变值均小于方案 1 。 翼缘处的应变也变得较为平缓, 说明设置辅助辊内外 实压板材, 使残余应力降低, 回弹量较少。

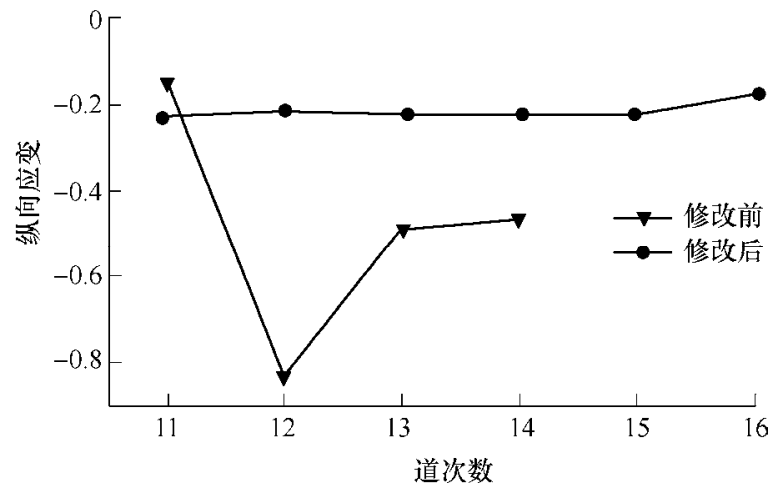

图 12 最大纵向应变对比

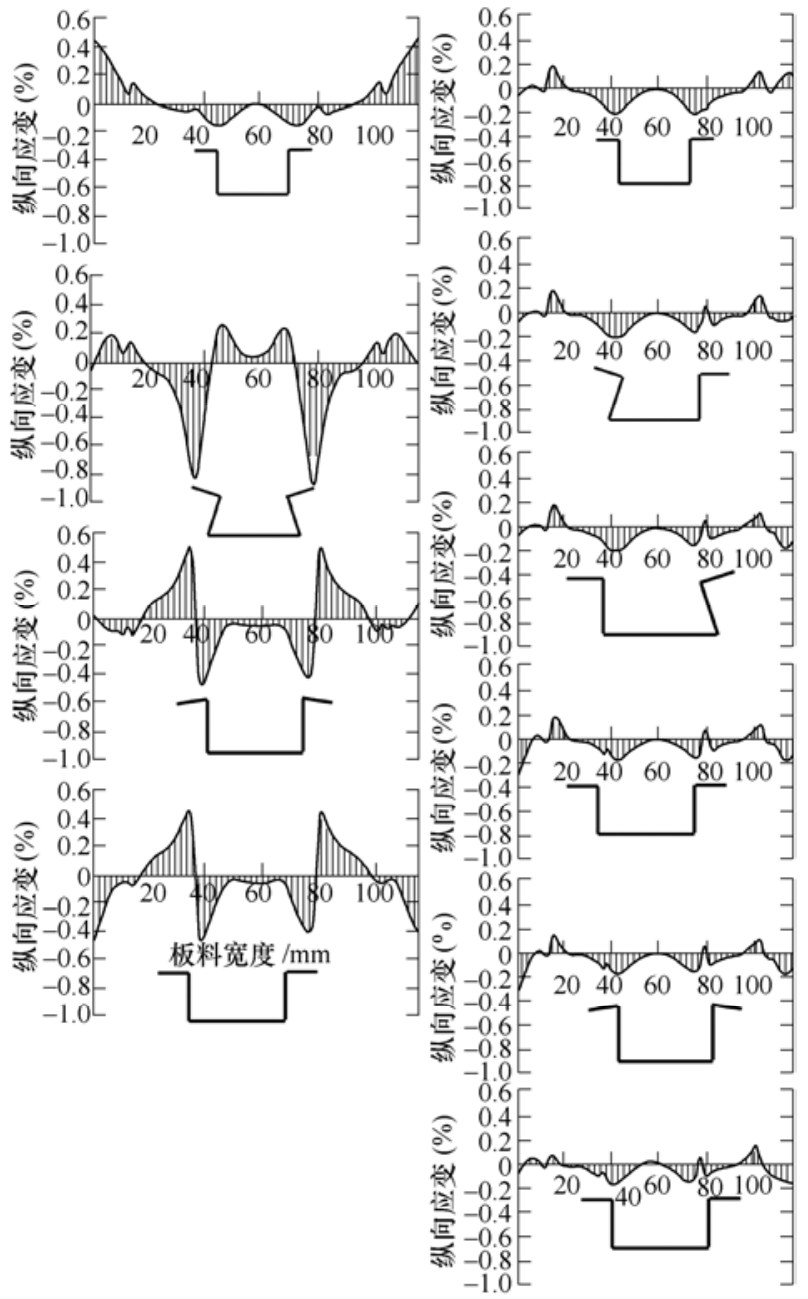

(a) 方案 1

(b) 方案 2

图 13 过弯道次纵向应变分布

\section{5 成形件截型精度分析}

将两个方案成形后的工件放在三维测量仪上, 在离入料端 150 mm(截面 1), 450 mm(截面 2), 750 $\mathrm{mm}$ (截面 3)处提取截面参考点(每个截面提取了 18 个参考点)的坐标, 如图 14、15 所示。

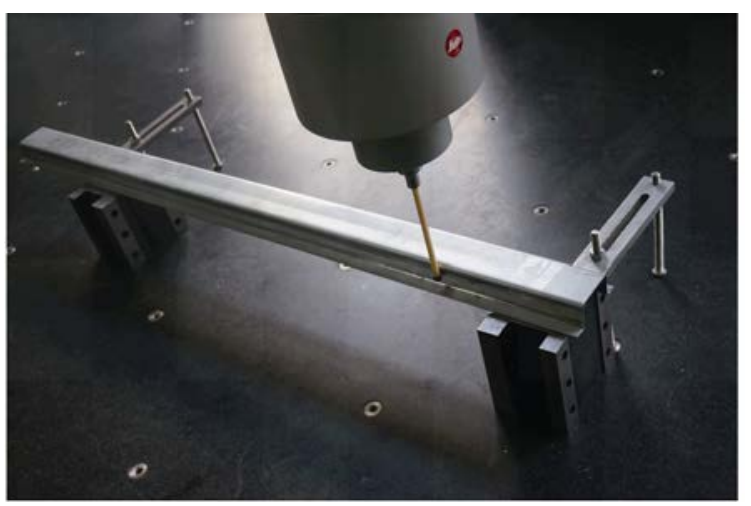

图 14 测量试验平台

观测空心点的分布, 可以看出修改辅辊以前, 位移偏移量最大的地方发生在板材右侧立边, 误差 


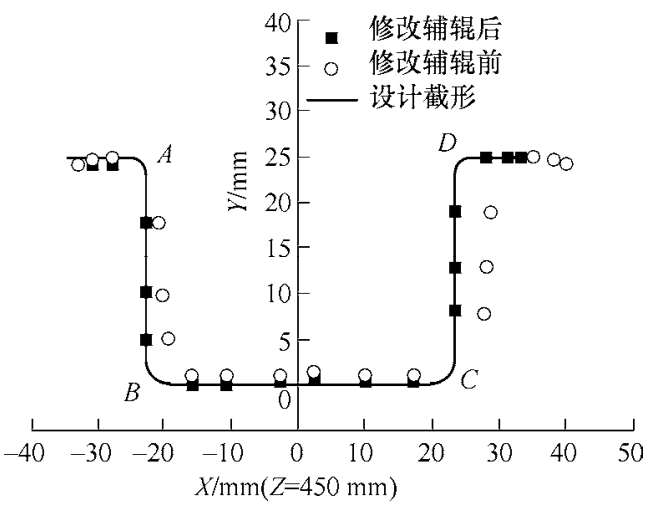

图 15 方案 1 和 2 截型精度对比

达到 $24.8 \%$, 且腹板处起拱明显, 整个成形件向右 侧扭曲, 翼缘处也有较大尧曲缺陷。说明在方案 1 的过弯辅辊设计下, 空弯使下弯角处材料受力不 均, 弯角处板材和轧辊接触变形剧烈, 产生了较大 的回弹。

辅辊修改后观测实心点分布, 通过和方案 1 对 比, 可知成形件截型回弹问题得到了一定程度地改 善。实心点分布更加接近设计截型, 各实测点的 $X / Y$ 向尺寸和设计尺寸对比偏移不大, 说明成形件没有 严重的缺陷，截型精度得到了提高。

成形件弯角回弹角度如表 4 所示, 可知方案 2 的过弯方式对改善下弯角回弹问题效果更佳, 成形 角度和设计截形的弯角误差均小于 $2 \%$ 。

表 4 轧辊修改前后弯角回弹量对比

\begin{tabular}{ccccc}
\hline 回弹角 & $\begin{array}{c}\text { 方案 } 1 \text { 结果/ } \\
\left({ }^{\circ}\right)\end{array}$ & $\begin{array}{c}\text { 误差 }(\theta \text { 或 } \delta) / 90 \\
(\%)\end{array}$ & $\begin{array}{c}\text { 方案 } 2 \text { 结果/ } \\
\left({ }^{\circ}\right)\end{array}$ & $\begin{array}{c}\text { 误差 }(\theta \text { 或 } \delta) / 90^{\circ} \\
(\%)\end{array}$ \\
\hline$A(\delta)$ & 0.67 & 2.26 & -0.31 & 0.34 \\
$B(\theta)$ & 0.67 & 8.18 & 0.32 & 0.35 \\
$C(\theta)$ & 0.67 & 7.83 & 1.49 & 1.66 \\
$D(\delta)$ & 0.67 & 1.44 & 0.67 & 0.74 \\
\hline
\end{tabular}

通过截型精度分析可知, 过弯辅辊设计会对产 品的最终截型精度产生影响, 空弯会使板材材料流 动不畅, 实弯设计更易于板材精准成形, 产品缺陷 也会更小。

\section{4 结论}

(1) 通过试验和仿真结果分析, 仿真模型和试 验结果所得数据的吻合度较好, 说明建立的帽型件 辊弯成形工艺有限元仿真模型能够有效地模拟成形 过程, 并且通过分析板材弯角处单元的应力应变场, 说明辊弯成形是一个反复加载-卸载的渐进过程, 在 成形过程中就出现弹性回复, 工件最终成形是多道 次总塑性应变累积的结果。

(2) 板材离开轧辊后, 弹性变形释放引起板材
的回弹，控制回弹问题的有效途径是增大变形过程 中的塑性变形，通常采用过弯进行回弹补偿，采用 外侧辅助辊过弯, 纵向阻力使材料发生进给方向上 的压应力, 由于下弯角内侧存在空弯, 导致下弯角 处材料流动不合理产生堆积，弯角变厚，不利于发 挥过弯的回弹补偿效果; 采用内外侧辅助辊过弯, 实弯使下弯角处的板材内外侧均与轧辊充分接触, 能有效增大弯角塑性变形区, 增大等效塑性应变值, 提高工件精度, 减少板材的弹性变形，使回弹问题 得到有效控制。

本文的研究结果对辊弯成形轧辊设计和板材纵 向应变预测均具有指导意义和参考价值。

\section{参 考 文 献}

[1] 韩飞, 刘继英, 艾正青, 等. 辊弯成型技术理论及应用 研究现状[J]. 塑性工程学报, 2010, 17(5): 71-78.

HAN Fei, LIU Jiying, AI Zhengqing, et al. State of the art of research on roll forming process[J]. Journal of Plasticity Engineering, 2010， 17(5): 71-78.

[2] PANTHI S K, RAMAKRISHNAN N, AHMED M, et al. Finite element analysis of sheet metal bending process to predict the springback[J]. Materials \& Design, 2010, 31(2): 657-662.

[3] 谢延敏, 孙新强, 田银, 等. 相变诱发塑性钢板的时效 回弹行为 [J]. 机械工程学报, 2014，52(6): 64-69.

XIE Yanmin, SUN Xinqiang, TIAN Yin, et al. Time-dependent springback behavior of transformationinduced plasticity steel[J]. Journal of Mechanical Engineering, 2014, 52(6): 64-69.

[4] XI Yang, CHANGHYOK C, NIMET K S, et al. Prediction of springback in air-bending of advanced high strength steel (DP780) considering Young's modulus variation and with a piecewise hardening function[J]. International Journal of Mechanical Sciences，2016， 105: 266-272.

[5] 余海燕, 王友. 一种基于 Chaboche 理论的混合硬化模 型及其在回弹仿真中的应用[J]. 机械工程学报, 2015, 51(16): 127-134.

YU Haiyan, WANG You. A combined hardening model based on Chaboche theory and its application in the springback simulation[J]. Journal of Mechanical Engineering, 2015, 51(16): 127-134.

[6] TENG Fei, ZHANG Wanxi, LIANG Jicai, et al. Springback prediction and optimization of variable stretch force trajectory in three-dimensional stretch bending process[J]. Chinese Journal of Mechanical Engineering, 2015, 28(6): 1132-1140.

[7] BUI Q V, PONTHOT J P. Numerical simulation of cold roll-forming processes[J]. Journal of materials processing 
technology, 2008, 202(1): 275-282.

[8] WEIBENGA J H, WEISS M, ROLFE B, et al. Product defect compensation by robust optimization of a cold roll forming process[J]. Journal of Materials Processing Technology, 2013(213): 978-986.

[9] JOHN P, KONSTANTINOS S , GEORGE C. Investigation of the effect of roll forming pass design on main redundant deformations on profiles from AHSS[J]. International Journal of Advanced Manufacturing Technology, 2011(56): 475-491

[10] SAFDARIAN R, NAEINI H M. The effect of forming parameters on the cold roll forming of channel section[J]. Thin-Walled Structures，2015，92: 130-136.

[11] 张宝, 丁武学, 彭斌涁. 下山法成形在辊弯成形中的应 用 $[\mathrm{J}]$. 锻压技术，2014，39(1)：52-57.
ZHANG Bao, DING Wuxue, PENG Binbin. Application of downhill method in roll forming[J]. Forging \& Stamping Technology, 2014, 39(1): 52-57.

[12] BIDABADI B S, NAEINI H M, TAFTI R A, et al. Experimental investigation of the ovality of holes on pre-notched channel products in the cold roll forming process[J]. Journal of Materials Processing Technology, 2015(225): 213-220.

[13] BIDABADI B S, NAEINI H M, TAFTI R A, et al. Experimental and numerical study of bowing defects in cold roll-formed, U-channel sections[J]. Journal of Constructional Steel Research, 2016(118): 243-253

作者简介(通信作者): 韩飞, 男, 1977 年出生, 硕士研究生导师。主要 研究方向为辊弯成形工艺及设备。

E-mail: hanfei@ncut.edu.cn

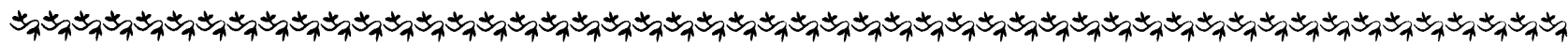

\section{(上接第 130 页)}

[13] PAKZAMAN H R, DIVANDARI M, KHAVANDI A R. Effect of nickel coating on steel wire reinforcement on mechanical properties of aluminum matrix composites produced via lost foam casting[C/CD]//Iran International Aluminum Conference, 2012.

[14] GÜLENÇ B, KAYA Y, DURGUTLU A, et al. Production of wire reinforced composite materials through explosive welding[J]. Archives of Civil \& Mechanical Engineering, 2016, 16(1): 1-8.

[15] SZCZEPANIK S. Composites of aluminium alloy matrix reinforced by a steel mesh[J]. Materials Today
Proceedings, 2015, 2: 9-18.

作者简介: 黄华贵(通信作者), 男, 1978 年出生, 博士, 教授, 博士研 究生导师。主要研究方向为层状金属复合材料制备工艺及装备、数值模 拟技术及工程应用。

E-mail: hhg@ysu.edu.cn

王计超, 男, 1993 年出生, 硕士研究生。主要研究方向为不锈钢丝网增 强铝基复合板轧制复合技术。

E-mail:2567214873@qq.com

刘文文, 男, 1990 年出生, 博士研究生。主要研究方向为层状金属复合 材料固-液铸轧复合技术。

E-mail: 837725878@qq.com

杜风山, 男, 1960 年出生, 教授, 博士研究生导师。主要研究方向为双 辊振动铸轧近终成形工艺与装备技术、轧机数字化技术。

E-mail: fsdu@ysu.edu.cn 